\title{
On the Use of Approximation Methods for Microcrack Shielding Problems
}

Department of Materials Science and Engineering, The Ohio State University, Columbus, $\mathrm{OH} 43210$

\author{
K. T. Faber \\ Department of Materials \\ Science and Engineering, \\ Northwestern University \\ Evanston, IL 60208
}

\begin{abstract}
There is experimental evidence that stress-induced microcracking near a macrocrack tip enhances the fracture toughness of brittle materials. In considering the interaction of the macrocrack with multiple microcracks using a discrete model, it is essential to use approximation methods in order to keep the amount of the computation to a tractable level. However, when crack distances are small, the results of the approximation methods can be significantly different from the numerical solution based upon the exact formulation. The results obtained by these approximation methods will be compared with the numerical solution to show the applicability ranges in which the errors are acceptably small. The use of results obtained by the approximation methods outside applicability ranges in literature is shown to lead to incorrect conclusions concerning microcrack shielding.
\end{abstract}

\section{Introduction}

There now exists experimental evidence that stress-induced microcracking near a macrocrack tip enhances the fracture toughness of brittle materials (Rühle, et al., 1987; Cai, et al., 1990; Faber, et al., 1990). Experimental studies by Rühle et al. (1987) have provided conclusive evidence of stress-induced microcracking toughening in a zirconia-toughened alumina. Recently, Faber et al. (1990) have shown a relationship between microcrack formation and an increase in toughness in $\mathrm{SiC}$ $\mathrm{TiB}_{2}$ composites with phases of different thermal expansion coefficient. The stress-induced microcracks near the macrocrack tip shield the macrocrack from the applied stress, thereby increasing the fracture toughness.

In addition to experimental studies, microcrack toughening has been also the subject of numerous modeling studies. The two basic approaches are continuum modeling (Evans and Faber, 1981; Clarke, 1984; Evans and Faber, 1984; Evans and Fu, 1985; Charalambides and McMeeking, 1987; Hutchinson, 1987; Ortiz, 1987; Charalambides and McMeeking, 1988; Laws and Brochenbrough, 1988; Ortiz and Giannakopoulos, 1989) and discrete modeling (Hoagland and Embury, 1980; Bowling, et al., 1987; Montagut and Kachanov, 1988). The continuum models are beyond the scope of this paper and will not be discussed further. Discrete models require consideration of the interaction of a macrocrack with microcracks (Kachanov and Montagut, 1986; Rose, 1986b, Rubinstein, 1986; Chudnovsky,

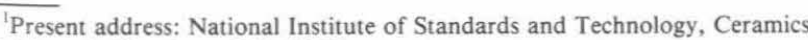
Division, Gaithersburg, MD 20899.

Contributed by the Applied Mechanics Division of The AMERICAN SocietY of Mechanical ENGINEers for publication in the ASME Journal of APpLIED MECHANICS.

Discussion on this paper should be addressed to the Technical Editor, Prof. Leon M. Keer, The Technological Institute, Northwestern University, Evanston, IL 60208 , and will be accepted until four months after final publication of the paper itself in the JOURNAL of APPLIED MEChanICS.

Manuscript received by the ASME Applied Mechanics Division, Mar. 30, 1990; final revision, Jan. 17, 1991. Associate Technical Editor: C. F. Shih
}

et al., 1987a; Chudnovsky, et al., 1987b; Hori and NematNasser, 1987; Rubinstein and Choi, 1988; Gong and Horii, 1989). For many microcracks necessary to treat the toughening problem, it is essential to use approximation methods to keep the amount of computation to a tractable level. Under certain conditions, the results of these approximation methods are very close to the exact solution. However, when the macrocrack-microcrack and microcrack-microcrack distances are small, the results of the approximation methods can be significantly different from the exact solution. Indiscriminate use of these results could inevitably lead to incorrect conclusions.

The purpose of this work is to evaluate three approximation methods using various microcrack configurations, and to estimate the range within which the approximation methods are applicable. Before doing so, an iterative method to solve the interaction between a macrocrack and an array of microcracks is described. Then, the solution obtained by the iterative method will be checked for the case of a collinear microcrack, for which the exact analytical solution is available.

\section{The Iterative Method}

The present approach is based upon the same principle of superposition and the concept of self-consistency applied to the interaction of cracks. An approximate solution based upon this method is the use of an average traction over each microcrack. An alternative approximation approach is the use of a point representation of microcracks (Hoagland and Embury, 1980; Rose, 1986b; Bowling, et al., 1987). We will present the solution in the context of a stationary macrocrack interacting with microcracks in the absence of residual stresses. The analysis is limited to two dimensions to keep the numerical computation tractable for the problem of a macrocrack interacting with many microcracks.

Consider a single microcrack of length $2 c$ and of arbitrary 

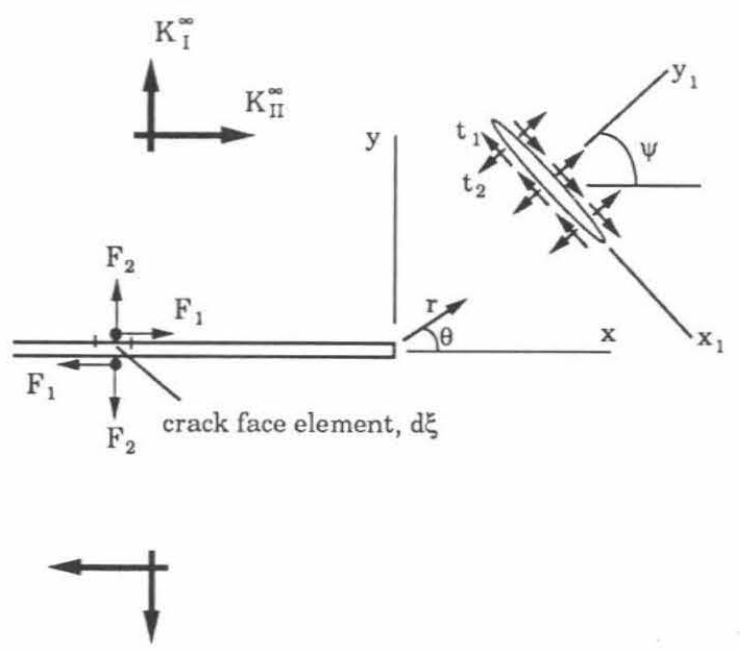

Fig. 1 Schematic of the main coordinate system and the microcrack coordinate system (adapted from Hoagland and Embury, 1980)

orientation near the crack tip of a semi-infinite crack with an associated applied stress intensity $\left(K_{I}^{\infty}\right.$ and $\left.K_{I I}^{\infty}\right)$ in two-dimensional space (Fig. 1). The near-tip stress field due to the applied stress without the microcrack is given by the following expression (Kanninen and Popelar, 1985):

$$
\sigma=K_{I}^{\infty} \sigma_{I}+K_{I I}^{\infty} \sigma_{I I}
$$

where $\sigma_{I}$ and $\sigma_{I I}$ are the modes I and II crack-tip stress fields given by

$\sigma_{I}=\frac{1}{\sqrt{2 \pi r}}\left(\begin{array}{ll}(\cos (\theta / 2)[1-\sin (\theta / 2) \sin (3 \theta / 2)] & \cos (\theta / 2) \sin (\theta / 2) \cos (3 \theta / 2) \\ \cos (\theta / 2) \sin (\theta / 2) \cos (3 \theta / 2) & \cos (\theta / 2)[1+\sin (\theta / 2) \sin (3 \theta / 2)]\end{array}\right)$

and

$\boldsymbol{\sigma}_{I I}=\frac{1}{\sqrt{2 \pi r}}\left(\begin{array}{ll}-\sin (\theta / 2)[2+\cos (\theta / 2) \cos (3 \theta / 3)] & \cos (\theta / 2)[1-\sin (\theta / 2) \sin (3 \theta / 2)] \\ \cos (\theta / 2)[1-\sin (\theta / 2) \sin (3 \theta / 2)] & \sin (\theta / 2) \cos (\theta / 2) \cos (3 \theta / 2)\end{array}\right)$.
The initial traction on the microcrack is given by

$$
\mathbf{t}=\mathbf{B} \cdot(\mathbf{n} \cdot \sigma)
$$

where $\mathbf{B}$ is the matrix of orthogonal transformation, $\mathbf{n}$ the unit normal of the microcrack, and $\sigma$ the existing stress along the microcrack line without the microcrack. The components of the stress are given in the main coordinate system, unless stated otherwise. The unit normal and the matrix of orthogonal transformation are related to the microcrack orientation angle $\psi$ by the following:

$$
\begin{gathered}
\mathbf{n}=\left(n_{1}, n_{2}\right)=(\cos \psi, \sin \psi), \\
\mathbf{B}=\left(\begin{array}{cc}
n_{2} & -n_{1} \\
n_{1} & n_{2}
\end{array}\right) .
\end{gathered}
$$

The stress field of a microcrack can be computed using Muskhelishvili formalism (Rice, 1968; Muskhelishvili, 1977). The appropriate line integral for the finite crack of length $2 c$ has been presented by Rice (1968), and is given as follows:

$$
\phi^{\prime}\left(z_{1}\right)=\frac{1}{2 \pi\left(z_{1}-c\right)^{1 / 2}\left(z_{1}+c\right)^{1 / 2}} \int_{-c}^{+c}\left[p_{2}(s)-i p_{1}(s)\right] \frac{\left(c^{2}-s^{2}\right)^{1 / 2}}{s-z_{1}} d s
$$

where $i$ is the imaginary unit, $z_{1}$ is the complex variable in the microcrack coordinate system and $z_{1}=x_{1}+i y_{1}$ (refer to Fig. $1), c$ is the half length of the microcrack, and $p_{i}=-t_{i}(s)$. The terms, $t_{1}(s)$ and $t_{2}(s)$ are components of $\mathbf{t}$, i.e., $\mathbf{t}(s)=$ $\left[t_{1}(s), t_{2}(s)\right]$. The stress components in the microcrack coordinate system are:

$$
\begin{gathered}
\sigma_{22}-i \sigma_{12}=\phi^{\prime}\left(z_{1}\right)+\phi^{\prime}\left(\bar{z}_{1}\right)+\left(z_{1}-\bar{z}_{1}\right) \bar{\phi}^{\prime \prime}\left(z_{1}\right) \\
\sigma_{11}=4 \operatorname{Re}\left[\phi^{\prime}\left(z_{1}\right)\right]-\sigma_{22}
\end{gathered}
$$

where Re indicates the real part of a complex number and a solid line over a complex number indicates its complex conjugate.

The stress field in the presence of a traction-free microcrack is the superposition of the stress field given by the equations listed above and the stress field without the microcrack. In other words, the microcrack stress when added to the exiting stress field produces a traction-free microcrack.

The microcrack stress field introduces tractions along the macrocrack face. To keep the macrocrack traction-free, an image stress field is introduced. The image stress field can be expressed in terms of a Green's function as derived by Hirth et al. (1974). The image stress at $z$ can be expressed in terms of line integrals

$$
\begin{gathered}
\sigma_{22}-i \sigma_{12}=\int_{-\infty}^{0}\left[\varphi^{\prime}(z)+\varphi^{\prime}(\bar{z})+(z-\bar{z}) \overline{\varphi^{\prime \prime}(z)}\right] d \xi \\
\sigma_{11}+\sigma_{22}=\int_{-\infty}^{0}\left[4 \operatorname{Re}\left[\varphi^{\prime}(z)\right]\right] d \xi
\end{gathered}
$$

where

$\varphi(z)=\frac{1}{2 \pi}\left[\sigma_{12}^{M}(\xi)+i \sigma_{22}^{M}(\xi)\right]\left[\ln \left(z^{1 / 2}+i|\xi|^{1 / 2}\right)-\ln \left(z^{1 / 2}-i|\xi|^{1 / 2}\right)\right]$,

and $\sigma_{i 2}^{M}(\xi)$ is the microcrack stress field computed along the macrocrack line. The integrals are evaluated numerically to find the image stress field. The image stress field is then su-

perposed onto the existing stress field. In doing so, new tractions are introduced to the once traction-free microcrack. These additional tractions are removed by applying Eqs. (6) and (7), and additional image stresses are computed using Eq. (8). The process is repeated until the tractions on the microcrack and the macrocrack are lower than a specified small value which varies (from $10^{-4}$ to $2 \mathrm{MPa}$ ) depending upon the accuracy required. The self-consistent stress field solution is then obtained.

The change in the stress intensity factor at the macrocrack tip is then computed:

$$
\begin{gathered}
\Delta K_{I}=\sqrt{(2 / \pi)} \int_{-\infty}^{0} \frac{\sigma_{22}^{M}(\xi)}{\sqrt{-\xi}} d \xi \\
\Delta K_{I I}=\sqrt{(2 / \pi)} \int_{-\infty}^{0} \frac{\sigma_{12}^{M}(\xi)}{\sqrt{-\xi}} d \xi .
\end{gathered}
$$

where $\sigma_{i j}^{M}(\xi)$ is the microcrack stress field evaluated along the macrocrack line. The integrals are evaluated numerically. The mode I stress intensity at the macrocrack tip can be written as

$$
K_{I}=K_{I}^{\infty}+\Delta K_{I} \text {. }
$$

Generally, this problem cannot be solved analytically, except in the case of collinear microcracks, the solutions of which have been presented by Rubinstein (1985) and Rose (1986a). Therefore, numerical methods are necessary to find the solution for a microcrack of arbitrary location and orientation. The numerical solution above does not represent a problem for the case of one or a few microcracks, but the computation 
becomes lengthy for multiple microcracks. The approximation methods which follow provide an alternative practical approach to the solution.

The first approximation method involves the use of average tractions on each microcrack, where the traction on a microcrack is averaged over the microcrack length. The stress field of a microcrack subject to a uniform surface traction can be simply expressed in terms of the Westergaard stress function (Westergaard, 1939; Sih, 1966; Eftis and Liebowitz, 1972). The appropriate stress function is

$$
Z\left(z_{1}\right)=\frac{1}{\sqrt{1-\left(c / z_{1}\right)^{2}}}-1
$$

where $z_{1}$ is the complex variable in the microcrack coordinate system. The microcrack stress field in the microcrack coordinate system can be expressed as

$$
\begin{gathered}
\sigma_{11}=t_{1}\left[2 \operatorname{Im} Z+y_{1} \operatorname{Re} Z,,_{z}\right]+t_{2}\left[\operatorname{Re} Z-y_{1} \operatorname{Im} Z,,_{z}\right] \\
\sigma_{22}=t_{1}\left[-y_{1} \operatorname{Re} Z,,_{z}\right]+t_{2}\left[\operatorname{Re} Z+y_{1} \operatorname{Im} Z, z\right] \\
\sigma_{12}=t_{1}\left[\operatorname{Re} Z-y_{1} \operatorname{Im} Z,,_{z}\right]+t_{2}\left[-y_{1} \operatorname{Re} Z,,_{z}\right]
\end{gathered}
$$

where $t_{1}$ is the shear component and $t_{2}$ is the normal component of traction $t$ on the microcrack surface, $Z,{ }_{z}$ is the first derivative of $Z$ with respect to $z_{1}$, and $\mathrm{Im}$ and $\mathrm{Re}$ indicate the imaginary part and the real part of a complex number, respectively.

Further simplification of the average traction method leads to another approximation method involving the use of a point representation of microcracks. In this approach, the traction on a microcrack is simply computed at the microcrack center. This approximation approach has been used in previous discrete modeling of microcrack shielding (Hoagland and Embury, 1980; Rose, 1986b; Bowling, et al., 1987).

\section{The Approximation Method by Kachanov and Mon-} tagut

Kachanov and Montagut (1986) used an approximation method to consider a semi-infinite crack and an array of microcracks. This method is based on the superposition technique and the ideas of self-consistency applied to the average tractions on individual cracks (or microcracks in the case of interaction of a macrocrack with $M$ microcracks). The stress field was represented as a superposition:

$$
\sigma(\mathbf{x})=K_{I} \sigma_{I}(\mathbf{x})+K_{I I} \sigma_{I I}(\mathbf{x})+\sum_{i=1}^{M} \sigma_{i}(\mathbf{x})
$$

where $\sigma_{I}$ and $\sigma_{I I}$ are the modes I and II asymptotic crack-tip fields given in Eq. (2), respectively, and $\sigma_{i}(\mathbf{x})$ is the stress field of $i$ th microcrack loaded by average traction $\left\langle\mathbf{t}_{i}\right\rangle$. The traction is induced along the microcrack line by other microcracks and the macrocrack stress field, and the average traction is given by

$$
\left\langle\mathbf{t}_{i}\right\rangle=K_{I} \mathbf{n}_{i} \cdot\left\langle\sigma_{I}\right\rangle_{i}+K_{I I} \mathbf{n}_{i} \cdot\left\langle\sigma_{I I}\right\rangle_{i}+\sum_{k} \boldsymbol{\Lambda}_{k i}\left\langle\mathbf{t}_{k}\right\rangle
$$

where $\mathbf{n}_{i}$ is the unit normal of $i$ th microcrack, $\left\langle\sigma_{I}\right\rangle_{i}$ and $\left\langle\sigma_{I I}\right\rangle_{i}$ are the average near-tip stress fields along the microcrack line, $\left\langle t_{k}\right\rangle_{i}$ is the average traction on the microcracks, and $\boldsymbol{\Lambda}_{k i}$ is the transmission factor (the average traction induced on $i$ th microcrack by $k$ th microcrack subject to unit traction).

There are $M$ vectorial unknowns, $\left\langle\mathbf{t}_{i}\right\rangle$, and two unknown scalars, $K_{I}$ and $K_{I I}$. With two additional conditions characterizing the effects of microcracks on the stress intensity of the macrocrack tip,

$$
K_{I}-K_{I}^{\infty}=\sqrt{(2 / \pi)} \int_{-\infty}^{0} \frac{1}{\sqrt{-\xi}} \sum_{i=1}^{M} \sigma_{(i) 22}^{M}(\xi) d \xi
$$

and

$$
K_{I I}-K_{I I}^{\infty}=\sqrt{(2 / \pi)} \int_{-\infty}^{0} \frac{1}{\sqrt{-\xi}} \sum_{i=1}^{M} \sigma_{(i) 12}^{M}(\xi) d \xi,
$$

(a)

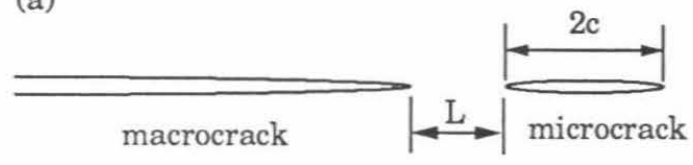

(b)

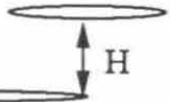

Fig. 2 Microcrack configurations used to evaluate the approximation methods

Table 1 Comparison of the results of the stress intensity by the numerical iterative method and the analytical solution

\begin{tabular}{llll}
\hline & \multicolumn{3}{c}{$\left(\mathrm{K}_{1}-\mathrm{K}_{\mathrm{I}}^{\infty}\right) / \mathrm{K}_{\mathrm{I}}^{\infty}$} \\
$\mathrm{L} / 2 \mathrm{c}$ & Numerical & Analytical & Error (\%) \\
\hline & & & \\
0.05 & 0.6497 & 0.6539 & 0.65 \\
0.10 & 0.3859 & 0.3873 & 0.40 \\
0.15 & 0.2729 & 0.2737 & 0.31 \\
0.20 & 0.2087 & 0.2092 & 0.26 \\
0.25 & 0.1671 & 0.1675 & 0.23 \\
0.30 & 0.1379 & 0.1382 & 0.21 \\
0.35 & 0.1164 & 0.1166 & 0.20 \\
0.40 & 0.0999 & 0.1001 & 0.19 \\
0.45 & 0.0870 & 0.0871 & 0.17 \\
0.50 & 0.0765 & 0.0766 & 0.17 \\
\hline
\end{tabular}

all of the unknowns can be evaluated. In Eq. (16), $K_{I}$ and $K_{I I}$ are the stress intensities at the macrocrack tip; $K_{I}^{\infty}$ and $K_{I I}^{\infty}$ are the applied stress intensities, and $\sigma_{(i) i j}^{M}(\xi)$ is the stress of $i$ th microcrack evaluated along the macrocrack line.

\section{Verification of the Numerical Solution}

The numerical solution (based upon the exact formulation) obtained by the iterative method for a collinear microcrack of length $2 c$ ahead of a macrocrack with an associated applied stress intensity of $K_{I}^{\infty}$ (Fig. 2(a)) is verified by comparing it to the analytical solution presented by Rubinstein (1985) and Rose (1986a). We consider a range of distances, $L$, between the microcrack tip and the macrocrack tip from 0.05 to 0.5 , that is, the macrocrack-microcrack tip distance is $1 / 20$ to $1 / 2$ of the microcrack length. The stress intensity at the macrocrack tip and the stress field at various points were computed. The results of the stress intensity are compared with values computed from the analytical solution in Table 1 . The maximum relative error in the range considered is 0.65 percent which is likely due to numerical computation (see Table 1). Better results can be obtained by refinement of the numerical computation.

\section{Evaluation of Approximation Methods}

The approximation methods: the iterative method with average traction (iterative-average), the iterative method with point representation of microcracks (iterative-point) and the approximation method by Kachanov and Montagut (1986) are compared with the numerical solution for certain microcrack orientations. The main emphasis is placed upon the mode I stress intensity, as mode I shielding is of particular interest.

To see the range within which the approximation methods are applicable, the distance between the macrocrack and microcrack is varied and the results obtained are compared. The two configurations considered are a collinear microcrack (Fig. 2(a)) and a horizontal microcrack parallel to the macrocrack 


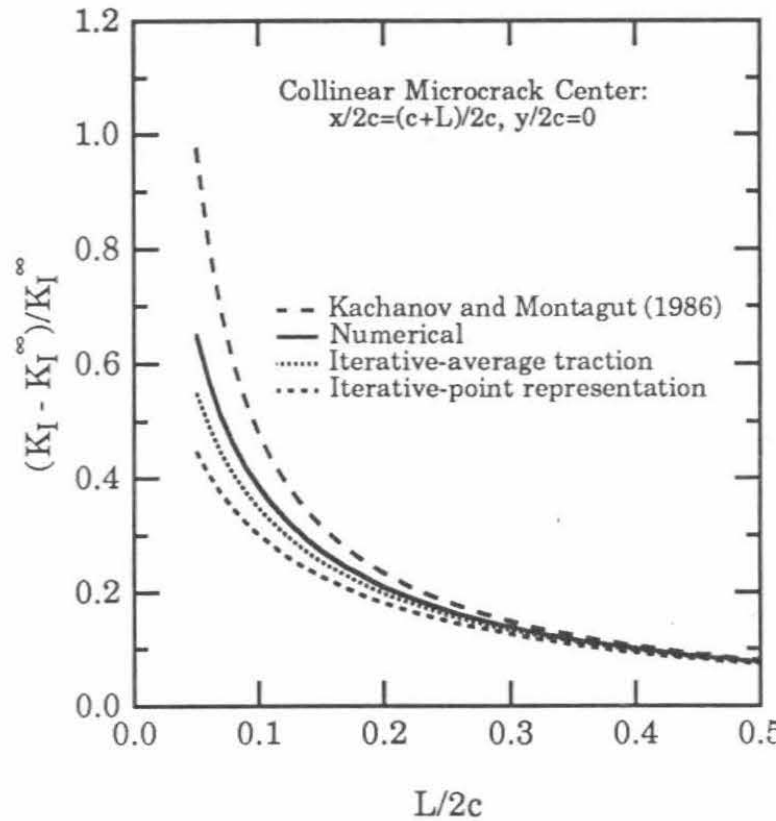

Fig. 3 Comparison of the change in the mode I stress intensity as a function of the normalized macrocrack-microcrack distance computed using the numerical solution, iterative-average traction, iterative-point representation, and the approximation method by Kachanov and Mon tagut for the collinear microcrack shown in Fig. 2(a).

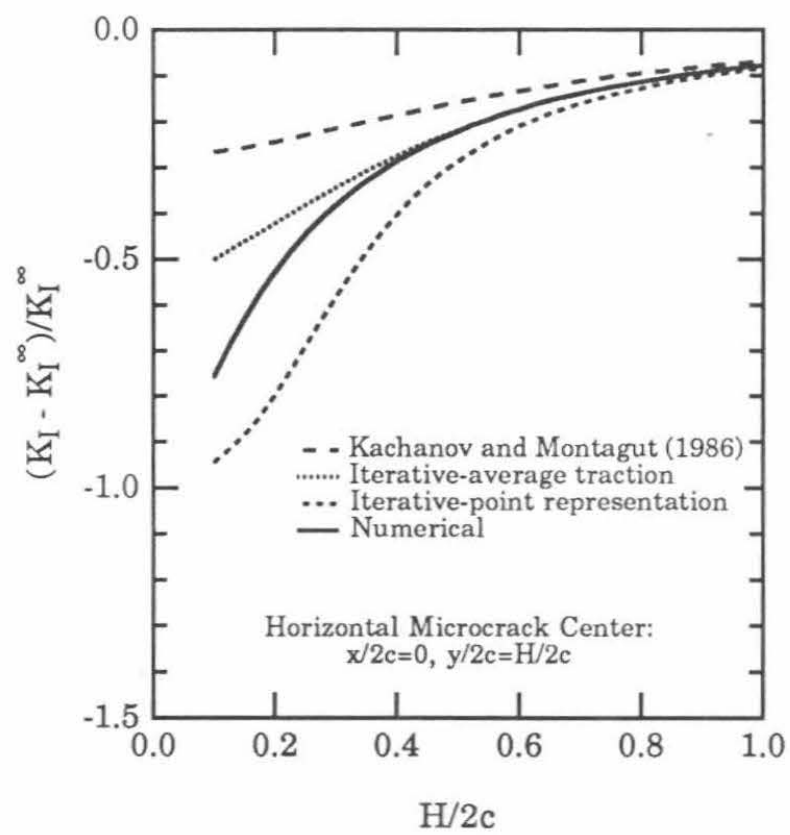

Fig. 4 Comparison of the change in the mode I stress intensity as a function of the normalized macrocrack-microcrack distance computed using the numerical solution, iterative-average traction, iterative-point representation, and the approximation method by Kachanov and Mon. tagut for the horizontal microcrack shown in Fig. 2(b)

and centered just above the macrocrack tip (Fig. 2(b)). Comparisons of the results are shown in Figs. 3 and 4 for the collinear microcrack and the horizontal microcrack cases, respectively. For the collinear microcrack as shown in Fig. 3, the results obtained by the approximation method by Kachanov and Montagut represent overestimates of antishielding, while those by the iterative-average traction method and the iterativepoint representation method represent underestimates of antishielding. For the horizontal microcrack as shown in Fig. 4,
Table 2 Range of applicability for approximation methods

\begin{tabular}{lll}
\hline $\begin{array}{l}\text { Approximation } \\
\text { Method }\end{array}$ & \multicolumn{2}{c}{ Applicable Range } \\
& $\begin{array}{l}\text { Collinear } \\
\text { microcrack }\end{array}$ & $\begin{array}{l}\text { Horizontal } \\
\text { microcrack }\end{array}$ \\
\hline Iterative-average & $\mathrm{L} / 2 \mathrm{c}>0.1$ & $\mathrm{H} / 2 \mathrm{c}>0.3$ \\
Iterative-point & $\mathrm{L} / 2 \mathrm{c}>0.3$ & $\mathrm{H} / 2 \mathrm{c}>0.9$ \\
Kachanov-Montagut & $\mathrm{L} / 2 \mathrm{c}>0.2$ & $\mathrm{H} / 2 \mathrm{c}>1.0$
\end{tabular}

the results obtained by the approximation method by Kachanova and Montagut represent undererstiamtes of shielding, while those by the iterative-point representation method represent overestimates of shielding. In both cases, the results obtained by the iterative-average traction method are closest to the numerical solution based upon the exact formulation. If we use the conventional definition of the relative error for the normalized change in stress intensity $\left(K_{I}-K_{I}^{\infty}\right) / K_{I}^{\infty}$, and arbitrarily determine that errors of ten percent or less are reasonable for the applicability of the approximation methods, we can compare the range within which the approximation methods are applicable for mode I stress intensities (see Table 2).

\section{Discussion}

In all the cases, the solutions obtained by approximation methods deviate from the exact solution as the distance between the macrocrack and microcrack decreases (Figs. 3 and 4). This is unfortunate because the microcracks nearest to the macrocrack tip have the greatest effects on the stress intensity of the macrocrack tip.

From Table 2, we can see that the iterative-average traction method offers the best accuracy among the three approximation methods discussed. With this method, the defined lowest range is $L / 2 c=0.1$ for the collinear microcrack case, and $H / 2 c=0.3$ for the horizontal microcrack case.

In the case of a collinear microcrack, the iterative method with point representation of microcracks underestimates the effect of the microcrack on the macrocrack; as a result, it underestimates antishielding (Fig. 3). In the case of a horizontal microcarck, it overestimates shielding (Fig. 4). Therefore, it tends to overestimate shielding.

In the case of the horizontal microcrack, the defined lower limit of applicability for the approximation method by Kachanov and Montagut is unexpectedly high. On the other hand, the defined lower limit of applicability is as low as $L /$ $2 c=0.2$ for the case of collinear microcrack. This favorable configuration of a collinear microcrack was used as the test case for the approximation method by Kachanov and Montagut (1986), and it was concluded that the error remains small for $L / 2 c$ as small as 0.1 to 0.2 . This conclusion does not hold for the case of the horizontal microcrack.

In the approximation method by Kachanov and Montagut, two approximations are involved in solving for the macrocrackmicrocrack interaction. The first is the use of the average traction induced on a microcrack. The second approximation is the use of the near-tip stress field in computing the superposed stress field (Eq. (14)). Consider the strong interaction of a macrocrack with a microcrack close to the macrocrack tip. In this case, the superposed stress field obtained by the second approximation is only good for $|\mathbf{x}|<<\left|\mathbf{x}_{m}\right|$, where $\mathbf{x}_{m}$ is the coordinate of the microcrack. Consequently, the use of this approximation in computing the traction on the microcrack is not good because $|\mathbf{x}| \approx\left|\mathbf{x}_{m}\right|$.

In the particular configuration of collinear microcrack in the Kachanov-Montagut treatment, the use of the average traction would results in an underestimate for the change in the stress intensity. On the other hand, the use of the increased 
stress intensity value at the macrocrack tip $\left(K_{I}\right)$ to compute the effect of the macrocrack on the microcrack (refer to Eq. (14)) leads to an overestimate. This overestimate is, in part, compensated by the underestimate due to the use of the average traction. With this compensation, the results obtained by the approximation method by Kachanov and Montagut method turn out to be good for $L / 2 c$ as low as 0.1 to 0.2 , as shown in Fig. 3.

For the horizontal microcrack in the Kachanov-Montagut treatment, the use of the average traction underestimates the change in the stress intensity. At the same time, the use of the reduced stress intensity value at the macrocrack tip $\left(K_{I}\right)$ to compute the effect of macrocrack on the microcrack also results in an underestimate. With this compounded underestimate, the results obtained by this method deviate from the exact solution quickly as $H / 2 c$ becomes small as shown in Fig. 4. We have observed that, in many cases, the approximation method by Kachanov and Montagut results in overestimates of antishielding, and underestimates of shielding, a feature also observed by other researchers (Rubinstein and Choi, 1988). This could explain, in part, why Kachanov and Montagut did not predict appreciable shielding from microcracks (Kachanov and Montagut, 1986; Montagut and Kachanov, 1988).

\section{Summary}

We have compared the results by the approximation methods with the numerical solution based upon the exact formulation for a number of cases. The following summary can be made: (1) Approximation methods should be applied with caution when the crack distance is small. Outside the applicable range, the results are misleading.

(2) Among the three approximation methods discussed, the iterative method with average tractions generally offers the best results.

(3) In many cases, the approximation method by Kachanov and Montagut overestimates antishielding, and underestimates shielding when the macrocrack-microcrack distance is small.

\section{Acknowledgment}

Support of this work has been provided by the National Science Foundation under Grant No. DMR-8896212.

\section{References}

Bowling, G. D., Faber, K. T., and Hoagland, R. G., 1987, "Computer Simulations of $R$-Curve Behavior in Microcracking Materials," J. Am. Ceram. Soc., Vol. 70, pp. 849-854

Cai, H., Gu, W.-H., and Faber, K. T., 1990, "Microcrack Toughening in a $\mathrm{SiC}-\mathrm{TiB}_{2}$ Composite,"' Proceedings of the American Society for Composites, Fifth Technical Conference on Composite Materials in Transition, Technomic Publishing Co., Lancaster, PA, pp. 892-901.

Charalambides, P. G., and McMeeking, R. M., 1987, “Finite Element Method Simulation of Crack Propagation in a Brittle Microcracking Solid," Mech. of Mater., Vol. 6, pp. 71-87.

Charalambides, P. G., and McMeeking, R. M., 1988, "Near-Tip Mechanics of Stress-Induced Microcracking in Brittle Materials," J. Am. Ceram. Soc., Vol. 71 , pp. $465-472$.
Chudnovsky, A., Dolgopolsky, A., and Kachanov, M., 1987a, "Elastic Interaction of a Crack with a Microcrack Array-I. Formulation of the Problem and General Form of the Solution,"'Int. J. Solids Struct., Vol. 23, pp. 1-10.

Chudnovsky, A., Dolgopolsky, A., and Kachanov, M., 1987b, "Elastic Interaction of a Crack with a Microcrack Array-II. Elastic Solution for Two Crack Configurations (Piecewise Constant and Linear Approximations)," Int. J. Solids Struct., Vol. 23, pp. 11-21.

Clarke, D. R., 1984, "A Simple Calculation of Process-Zone Toughening by Microcracking," J. Am. Ceram. Soc., Vol. 67, pp. C15-C16.

Eftis, J., and Liebowitz, H., 1972, "On the Modified Westergaard Equations for Certain Plane Crack Problems,” Int. J. Fract. Mech., Vol. 8, pp. 383-392.

Evans, A. G., and Faber, K. T., 1981, "Toughening of Ceramics by Circumferential Microcracking," J. Am. Ceram. Soc., Vol. 64, pp. 394-398.

Evans, A. G., and Faber, K. T., 1984, "Crack-Growth Resistance of Microcracking Brittle Materials,” J. Am. Ceram. Soc., Vol. 67, pp. 255-260.

Evans, A. G., and Fu, Y., 1985, "Some Effects of Microcracks on the Mechanical Properties of Brittle Solids-II. Microcrack Toughening,"'Acta Metall., Vol. 33, pp. 1525-1531.

Faber, K. T., Gu, W.-H., Cai, H., Winholtz, R. A., and Magley, D. J., 1991, "Fracture Properties of SiC-Based Particulate Composites," Proceedings of the NATO Advanced Research Workshop on Toughening Mechanisms in QuasiBrittle Materials, Kluwer Academic Publishers, The Netherlands, pp. 3-17.

Gong, S.-X., and Horri, H., 1989, "General Solution to the Problem of Microcracks Near the Tip of a Main Crack," J. Mech. Phys. Solids, Vol. 37, pp. $27-46$.

Hirth, J. P., Hoagland, R. G., and Gehlen, P. C., 1974, "The Interaction Between Line Force Arrays and Planar Cracks," Int. J. Solids Struct., Vol. 10, pp. 977-984.

Hoagland, R. G., and Embury, J. D., 1980, "A Treatment of Inelastic Deformation Around a Crack Tip due to Microcracking," J. Am. Ceram. Soc., Vol. 63 , pp. $404-410$.

Hori, M., and Nemat-Nasser, S., 1987, "Interacting Micro-Cracks Near the Tip in the Process Zone of a Macro-Crack," J. Mech. Phys. Solids, Vol. 35, pp. 601-629.

Hutchinson, J. W., 1987, "Crack Tip Shielding by Micro-Cracking in Brittle

Solids," Acta Metall., Vol. 35, pp. 1605-1619.

Kachanov, M., and Montagut, E., 1986, "Interaction of a Crack with Certain Microcrack Arrays," Eng. Fract. Mech., Vol. 25, pp. 625-636.

Kanninen, M. F., and Popelar, C. H., 1985, Advanced Fracture Mechanics, Oxford University Press, New York.

Laws, N., and Brockenbrough, J. R., 1988, "Microcracking in Polycrystalline Solids," J. Eng. Mater. Technol., Vol. 110, pp. 101-104.

Montagut, E., and Kachanov, M., 1988, "On Modelling a Microcracked Zone by “Weakened"' Elastic Material and On Statistical Aspects of Crack-Microcrack Interactions," Int. J. Fract., Vol. 37, pp. R55-R62.

Muskhelishvili, N. I., 1977, Some Basic Problems in the Mathematical Theory of Elasticity, 2nd English ed., Noordhoff International Publishing, Leyden, The Netherlands.

Ortiz, M., 1987, "A Continuum Theory of Crack Shielding in Ceramics," ASME Journal of APplied Mechanics, Vol. 54, pp. 54-58.

Ortiz, M., and Giannakopoulos, A. E., 1989, "Maximal Crack Tip Shielding by Microcracking,"' ASME Journal of Applied Mechanics, Vol. 56, pp. 279283.

Rice, J. R., 1968, "Mathematical Analysis in the Mechanics of Fracture," Fracture, Vol. II, H. Liebowitz, ed., Academic Press, New York, pp. 191-311.

Rose, L. R. F., 1986a, "Effective Fracture Toughness of Microcracked Materials,” J. Am. Ceram. Soc., Vol. 69, pp. 212-214.

Rose, L. R. F., 1986b, "Microcrack Interaction with a Main Crack," Int. J. Fract., Vol. 31, pp. 233-242.

Rubinstein, A. A., 1985, "Macrocrack Interaction with Semi-infinite Microcrack Array,"'Int. J. Fract., Vol. 27, pp. 113-119.

Rubinstein, A. A., 1986, "Macrocrack-Microdefect Interaction," ASME Journal of Applied Mechanics, Vol. 53, pp. 505-510.

Rubinstein, A. A., and Choi, H. C., 1988, "Macrocrack Interaction with Transverse Array of Microcracks,"' Int. J. Fract., Vol. 36, pp. 15-26.

Rühle, M., Evans, A. G., McMeeking, R. M., Charalambides, P. G., and Hutchinson, J. W., 1987, "Microcrack Toughening in Alumina/Zirconia," Acta Metall., Vol. 35, pp. 2701-2710.

Sih, G. C., 1966, "On the Westergaard Method of Crack Analysis," Int. J. Fract. Mech., Vol. 2, pp. 628-631.

Westergaard, H. M., 1939, "'Bearing Pressures and Cracks," ASME JournaI of Applied Mechanics, Vol. 61, pp. A49-A53. 